\title{
Crecimiento de larvas de mosca soldado alimentadas con gallinaza, porcinaza y alimento para ponedoras
}

Growth of larvae of the soldier fly fed with hen manure, swine manure and layer poultry fed

Arroyave-Sierra OJ, Chamorro-Rengifo J, Ochoa-Muñoz AF. Crecimiento de larvas de mosca soldado alimentadas con gallinaza, porcinaza y alimento para ponedoras. Rev Colombiana Cienc Anim. Recia. 2019; 11(2):Articulo730. DOI: https://doi.org/10.24188/recia.v11.n1.2019.730

Universidad de Sucre, Colombia

Los autores permiten a RECIA reimprimir el material publicado en él. En caso de que un autor quiera traducir o usar una publicación parcial o completa de nuestro Diario, el autor debe obtener un permiso por escrito del editor de la revista.

Revista Colombiana de Ciencia Animal - RECIA está distribuido bajo una Licencia Creative Commons Atribución-CompartirIgual 4.0 Internacional. 


\title{
Crecimiento de larvas de mosca soldado alimentadas con gallinaza, porcinaza $\mathrm{y}$ alimento para ponedoras
}

\author{
Growth of larvae of the soldier fly fed with hen manure, swine manure and layer \\ poultry fed
}

Oscar Julián Arroyave Sierra, M.Sc.

DOI: https://doi.org/10.24188/recia.v11.n2.2019.730

Centro de Biotecnología Industrial - SENA Palmira

oscarj.arroyave@sena.edu.co

(D) https://orcid.org/0000-0002-3152-3707

Juliana Chamorro Rengifo, Ph.D.

Centro de Biotecnología Industrial - SENA Palmira. IE José María

Villegas. Buga

julianachamorro@gmail.com

(D) https://orcid.org/0000-0003-0707-8268

Andrés Felipe Ochoa Muñoz, M.Sc.

Centro de Biotecnología Industrial - SENA Palmira.

Universidad del Valle, Facultad de Ingeniería, Escuela de

Estadística, Cali.

andresochoa7788@gmail.com

(D) https://orcid.org/0000-0002-0003-1347

Recepción: 30 Julio 2019

Aprobación: 9 Noviembre 2019

Publicación: 12 Noviembre 2019

\section{RESUMEN}

En esta investigación se estudió el efecto de la gallinaza, porcinaza y alimento para ponedoras, sobre el crecimiento de larvas de mosca soldado; este último evaluado a través del cambio del peso, ancho y largo de las larvas. Los datos fueron analizados mediante modelos mixtos de coeficientes aleatorios, puesto que las variables fueron analizadas en el tiempo y se producen datos longitudinales. Se analizó el crecimiento de las larvas durante cuatro semanas y se observó que la alimentación con porcinaza produce larvas con menor desempeño en peso, largo y ancho, comparado con las alimentadas con gallinaza y alimento para ponedoras, los cuales no presentan diferencias significativas, lo que indica que la gallinaza y el alimento para ponedoras son más eficientes en términos de crecimiento. Es importante seguir realizando experimentos que evidencien que la gallinaza y el alimento para ponedoras son más apropiados para el crecimiento de las larvas de mosca soldado.

Palabras clave: datos longitudinales, Hermetia illucens, morfometría, reciclaje, residuos orgánicos.

\begin{abstract}
This research studied the effect of three diets, hen manure, swine manure and poultry feed, on the growth of soldier fly larvae, based on the change in the variables weight, width and length of the larvae. The data were analyzed using mixed models of random coefficients, since variables were analyzed over time and longitudinal data are produced. The growth of larvae was analyzed in four weeks and it was observed that feeding with swine manure produces larvae with lower performance in weight, length and width, compared to hen manure and layer feed, which are not significantly different, indicating that hen manure and layer feed are more efficient in terms of growth. It is important to continue to conduct experiments that show that hen manure and layer feed are more appropriate for the growth of soldier fly larvae
\end{abstract}

Keywords: Hermetia illucens, longitudinal data, morphometry, organic waste, recycling 


\section{INTRODUCCIÓN}

Se prevé que la población mundial aumentará más de un tercio de las personas entre el 2009 y el 2050. Las proyecciones muestran que para alimentar una población mundial de 9100 millones de habitantes en el 2050 sería necesario aumentar la producción de alimentos en un 70\%. La producción en los países en desarrollo tendrá que duplicarse. Esto significa un aumento importante en la producción de varios productos básicos fundamentales. La producción de cereales, por ejemplo, tendrá que incrementarse en casi mil millones de toneladas, y la producción de carne en más 200 millones de toneladas, hasta alcanzar 470 millones de toneladas en el 2050 - el 72\% de estas en los países en desarrollo frente al 58\% de la actualidad. - Alimentar de forma adecuada toda esa población mundial significará producir los tipos de alimentos que faltan para garantizar la seguridad nutricional (1).

La tendencia mundial hacia la intensificación de la producción en grandes unidades de producción pecuaria causa la masiva producción y acumulación de estiércol. El manejo inadecuado del estiércol producido por la operación de estas granjas industriales deteriora en gran medida los principales medio ambientales, aire, agua y suelos (2).

La mosca soldado, Hermetia illucens L. (Diptera, Stratiomyidae) es nativa del Neotrópico y actualmente se encuentra distribuida ampliamente por casi todas las áreas cálidas del mundo. En las últimas décadas ha habido un considerable interés en utilizar larvas de H. illucens para el control de desechos orgánicos, procesamiento de compostaje y como suplemento alimenticio para animales (3). Las larvas son polífagas y se pueden alimentar de una gran variedad de fuentes (4). Además, se utilizan como alimento para animales debido a su valioso perfil nutricional. En promedio, las larvas consisten en $40 \%$ de proteína y 35\% de grasa (5). Estudios en donde se utilizaron larvas de $H$. illucens como parte de piensos para pollos, cerdos, bagres y tilapia han demostrado resultados positivos $(6,7,8$, 9). Además, el reciclaje de desechos agrícolas en forma de alimento reduce considerablemente la contaminación orgánica (10). Es por eso que, en criaderos de pollos y cerdos, se podrían utilizar las larvas de mosca soldado para procesar los desechos orgánicos (gallinaza y porcinaza) a la vez que se pueden utilizar como alimento para los animales.

Se denomina gallinaza a los desechos sólidos de la producción de gallina ponedora, compuesta por la cama o sin ésta, la excreta y demás residuos como restos de alimentos, huevos rotos y plumas (11). La gallinaza debe ser tratada antes de ser movilizada o comercializada, para minimizar el riesgo sanitario y/o evitar transmisión de agentes patógenos (12). La porcinaza es la mezcla de heces y orina de los cerdos, con descamaciones, pelo, comida no digerida y cantidades variables de agua, y en algunos casos contiene material de origen vegetal usado como cama. Al igual que la gallinaza, la porcinaza debe ser procesada y modificada para disminuir los riesgos de contaminación ambiental. Tanto la gallinaza como la porcinaza procesada pueden ser utilizados como fertilizantes de suelos y cultivos, para producir energía (biogás) y como alimento (13).

El presente trabajo tuvo como objetivo evaluar el efecto del uso de gallinaza y porcinaza como alimento, sobre el crecimiento de las larvas de H. illucens.

\section{MATERIALES Y MÉTODOS}

Cría, alimentación de las larvas y registro de variables. El experimento se realizó en las instalaciones del Centro de Biotecnología Industrial, CBI-SENA en Palmira Valle del Cauca, Colombia. Se establecieron tres tratamientos con tres repeticiones cada uno para un total de 9 unidades experimentales, cada unidad experimental consistía en un recipiente plástico capacidad 1 kilo, con tapa modificada para permitir la respiración de las larvas. Se introdujeron 100 larvas de 5 días de edad obtenidas en la unidad de producción de mosca soldado del Centro Latinoamericano de Especies Menores, CLEM SENA- Tuluá. A cada grupo se le asignó un tipo de dieta, gallinaza o porcinaza, y se utilizó como control, alimento marca comercial para ponderas. Cada porción de alimento consistió en 200 gr al 70\% de humedad por una sola vez. Para evaluar la respuesta del crecimiento a las dietas, se midió el peso (gr) en balanza digital, ancho (mm) y largo (mm) con un micrómetro digital. Las mediciones fueron tomadas al 10\% de las larvas de cada repetición, al inicio, 5 - 9 - 12 - 14 días del periodo de engorde. Las larvas eran extraídas del sustrato, lavadas, medidas y pesadas, para luego volver a ser introducidas al recipiente.

Como los datos fueron analizados a lo largo del tiempo, se generaron datos longitudinales, los cuales se ajustaron por medio de modelos mixtos de coeficientes aleatorios (14).

Análisis Estadístico. Inicialmente se realizaron análisis descriptivos con el fin de conocer el comportamiento de las variables respuestas en función de los tratamientos y el tiempo. Se utilizaron diagramas de cajas con el fin de analizar 
la variabilidad e identificar observaciones atípicas. Además, se efectuaron tres modelos mixtos, uno para cada variable respuesta; estos modelos se ajustaron con el software R versión 3.5.1 y la librería "nlme" (15).

Para cada modelo se consideró el efecto asociado al tratamiento (alimento para ponedoras, gallinaza, porcinaza) y el efecto asociado al tiempo (semanas de medición). De esta manera, se planteó la expresión del modelo estadístico:

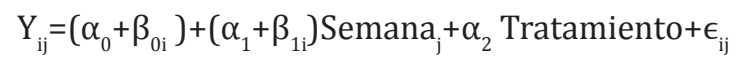

Donde $\mathrm{Y}_{\mathrm{ij}}$ es la variable respuesta de la larva i en la semana $\mathrm{j}$, donde $\mathrm{i}=1,2,3, . ., 90$ asociado a las observaciones y replicas; $\mathrm{j}=0,1,2,3,4$ asociado a las cuatro semanas en las cuales fueron realizadas las mediciones; $\alpha_{\mathrm{k}}=0,1,2$ son los coeficientes asociados a la parte fija del modelo. Los coeficientes $\beta_{0 \mathrm{i}} \mathrm{y} \beta_{1 \mathrm{i}}$ están asociados al intercepto y la pendiente aleatoria, y $\epsilon_{\mathrm{ij}}$ es un error aleatorio no controlable por la experimentación. Las estimaciones de los parámetros de varianza se realizaron a partir de procesos iterativos con el algoritmo Newton-Raphson. En los contrastes de hipótesis para los parámetros del modelo se utilizó un nivel de significancia del 5\%.

\section{RESULTADOS}

Análisis Descriptivo. Los datos indican que a medida que pasan las semanas, las larvas aumentan de peso (Figura 1). En particular, en la semana cuatro, gallinaza y alimento para ponedoras no presentaron pesos muy diferentes en cuanto a sus réplicas. También se observa una diferencia en el tratamiento porcinaza, siendo éste un tratamiento con mayor variabilidad en el peso. La Figura 1 se debe interpretar en conjunto con la modelación, puesto que se puede evaluar si existen diferencias significativas entre tratamientos a lo largo del tiempo.

El ancho de las larvas también aumentó al pasar las semanas (Figura 2). Para el tratamiento alimento para ponedoras, las larvas iniciaron con una mediana de $1 \mathrm{~mm}$ y al cabo de la cuarta semana, alcanzaron una mediana de $6 \mathrm{~mm}$. Por su parte, el tratamiento gallinaza inició con una mediana de $1 \mathrm{~mm}$ y llegó a la semana cuatro con una mediana de $5 \mathrm{~mm}$. Las larvas alimentadas con porcinaza presentaron un incremento menor en el ancho; inició con una mediana de 1 mm y alcanzaron a la cuarta semana una mediana de $2.2 \mathrm{~mm}$. Se concluye que el tratamiento alimento para ponedoras presentó mejor desempeño respecto a la variable ancho, seguido por los tratamientos gallinaza y porcinaza. Es importante evaluar con la modelación, si las diferencias en alimento para ponedoras son significativa al comparar con los demás tratamientos

La variable largo inició en $5 \mathrm{~mm}$ y aumentó a medida que pasaron las semanas, llegando a la cuarta semana con largos mayores a $20 \mathrm{~mm}$ en los tratamientos alimento para ponedoras y gallinaza. Para el tratamiento porcinaza, la mediana del largo fue de alrededor de los $9.5 \mathrm{~mm}$. En términos generales, los tratamientos alimento para ponedoras y gallinaza presentaron mejor desempeño para la variable largo (Figura 3).

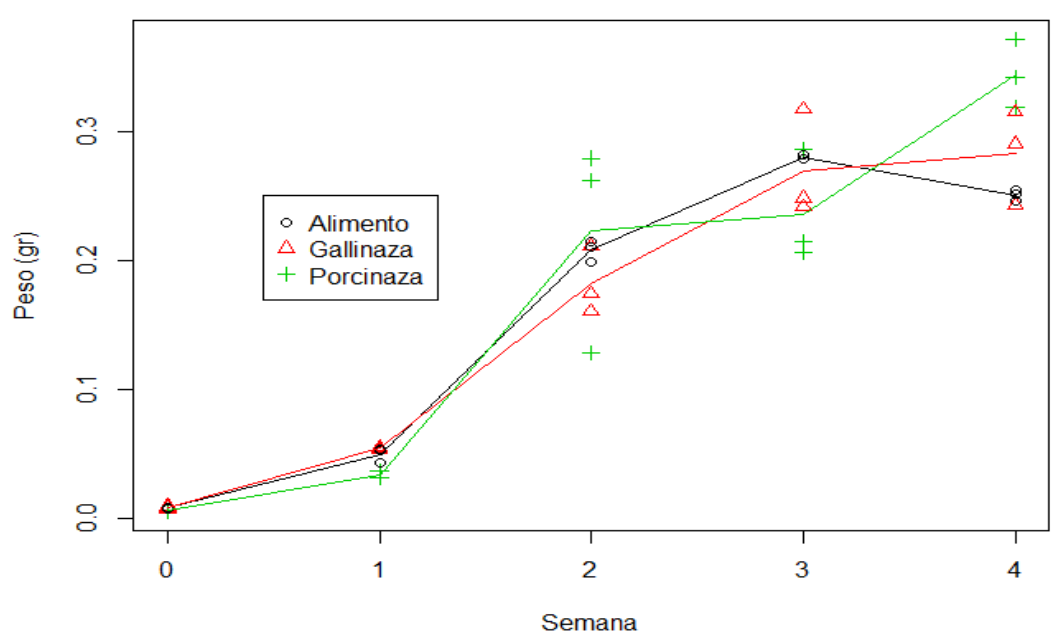

Figura 1. Comportamiento del peso por semana y tratamiento. 


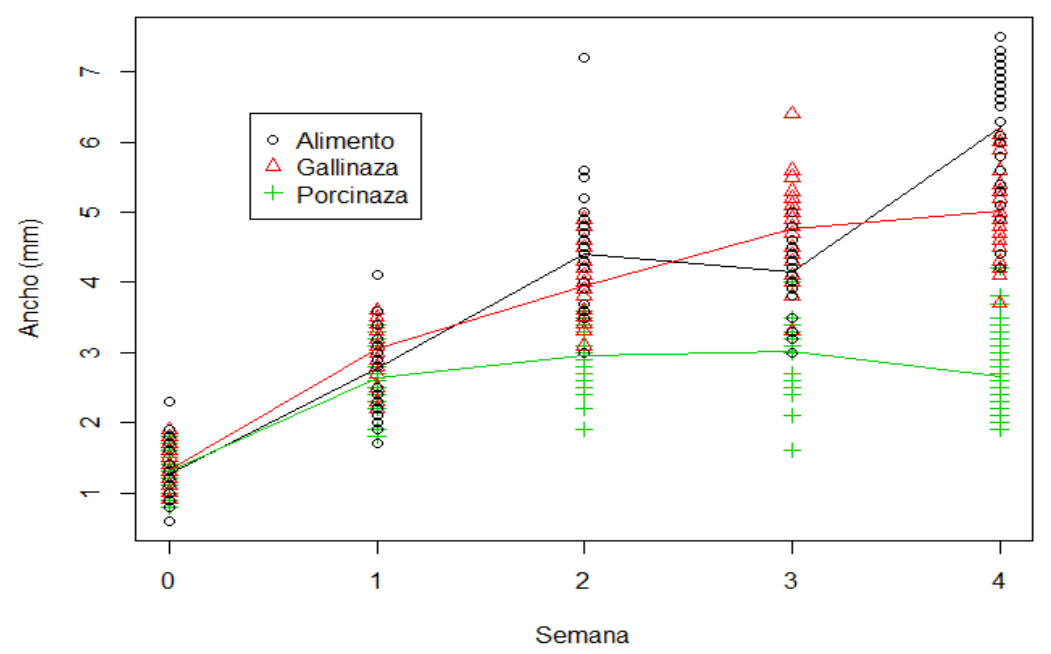

Figura 2. Comportamiento para el ancho por semana y tratamiento

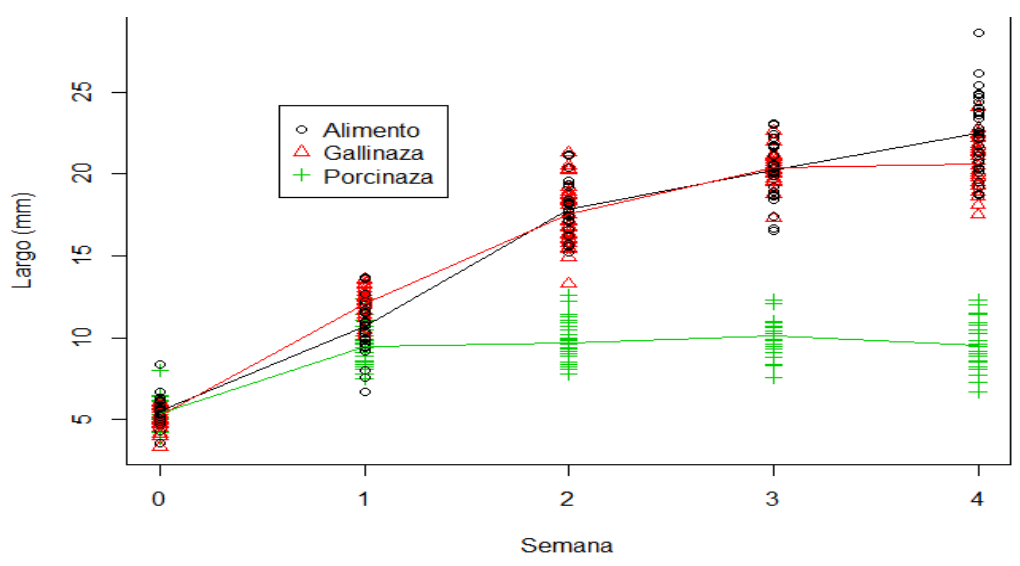

Figura 3. Comportamiento para el largo por semana y tratamiento

Modelos mixtos para el peso, ancho y largo. En el modelo para la variable peso, el factor asociado al tratamiento no es significativo, lo cual indica que el tipo de tratamiento no tiene una influencia en el peso de las larvas (ANOVA, Tabla 1). Por el contrario, las variables respuesta ancho y largo están influenciadas por el tipo de tratamiento. Por lo tanto, uno de los tratamientos presenta diferencias significativas con relación a las variables largo y ancho.

Tabla1. ANOVA en cada modelo mixto.

\begin{tabular}{|c|c|c|c|c|c|}
\hline Variable Respuesta & Parámetro & numDF & denDF & $\mathbf{F}$ & $P$ valor \\
\hline & Intercepto & 1 & 39 & 441.7711 & $<.0001$ \\
\hline \multirow[t]{3}{*}{ Peso } & Semana & 1 & 39 & 251.7748 & $<.0001$ \\
\hline & Tratamiento & 2 & 39 & 0.2033 & 0.8169 \\
\hline & Intercepto & 1 & 359 & 6855.380 & $<.0001$ \\
\hline \multirow[t]{3}{*}{ Ancho } & Semana & 1 & 359 & 770.683 & $<.0001$ \\
\hline & Tratamiento & 2 & 87 & 97.401 & $<.0001$ \\
\hline & Intercepto & 1 & 359 & 8570.248 & $<.0001$ \\
\hline \multirow[t]{2}{*}{ Largo } & Semana & 1 & 359 & 925.506 & $<.0001$ \\
\hline & Tratamiento & 2 & 87 & 227.903 & $<.0001$ \\
\hline
\end{tabular}


Para identificar el tratamiento que presenta efecto significativo en los modelos, se utilizó la estimación asociada a los efectos fijos de los modelos (Tabla 2). Para la variable peso, el factor asociado a la semana indica que a medida que pasan las semanas se aumenta el peso en un promedio de 0.0785 gr. Los tratamientos asociados a gallinaza y porcinaza no presentaron diferencias significativas al compararlos con el tratamiento alimento para ponedoras (ver también Tabla 1 del ANOVA).

Tabla 2. Estimación de los efectos fijos en cada modelo mixto.

\begin{tabular}{|c|c|c|c|c|c|c|}
\hline Variable Respuesta & Parámetro & Estimación & Error Estándar & DF & t-valor & p-valor \\
\hline \multirow{4}{*}{ Peso } & Intercepto & 0.0017 & 0.0151 & 39 & 0.1147 & 0.9093 \\
\hline & Semana & 0.0785 & 0.0049 & 39 & 15.8674 & 0.0000 \\
\hline & Tratamiento-Gallinaza & 0.0001 & 0.0165 & 39 & 0.0047 & 0.9962 \\
\hline & Tratamiento-Porcinaza & 0.0091 & 0.0165 & 39 & 0.5545 & 0.5823 \\
\hline \multirow{4}{*}{ Ancho } & Intercepto & 2.1966 & 0.0891 & 359 & 24.6496 & 0.0000 \\
\hline & Semana & 0.7823 & 0.0281 & 359 & 27.7611 & 0.0000 \\
\hline & Tratamiento-Gallinaza & -0.1406 & 0.0976 & 87 & -1.4409 & 0.1532 \\
\hline & Tratamiento-Porcinaza & -1.2440 & 0.0976 & 87 & -12.7431 & 0.0000 \\
\hline \multirow{4}{*}{ Largo } & Intercepto & 9.2637 & 0.3172 & 359 & 29.2028 & 0.0000 \\
\hline & Semana & 3.0517 & 0.1003 & 359 & 30.4221 & 0.0000 \\
\hline & Tratamiento-Gallinaza & -0.1860 & 0.3474 & 87 & -0.5352 & 0.5938 \\
\hline & Tratamiento-Porcinaza & -6.5160 & 0.3474 & 87 & -18.7511 & 0.0000 \\
\hline
\end{tabular}

En el modelo mixto para la variable respuesta ancho (Tabla 2) se presentan diferencias significativas para el tratamiento porcinaza, de tal forma que al pasar del tratamiento alimento para ponedoras al tratamiento porcinaza, hay una disminución del ancho de las larvas en promedio de $-1.24 \mathrm{~mm}$. El tratamiento gallinaza presentó resultados iguales a los del tratamiento alimento para ponedoras, debido a que dicho tratamiento no es significativo. Además, el factor asociado al tiempo indica que a medida que aumentan las semanas se aumenta el ancho de las larvas en un promedio de 0.7823 $\mathrm{mm}$; siendo el factor asociado a la semana significativo para el modelo.

El modelo mixto asociado al largo de la larva indica que el tratamiento porcinaza es significativamente diferente al tratamiento alimento para ponedoras, ya que disminuye el largo de la larva en un promedio de $-6.5160 \mathrm{~mm}$, por lo tanto, las larvas alimentadas con porcinaza presentan largo menor durante las semanas de evaluación (Tabla 2). Para el tratamiento gallinaza no se encontraron diferencias significativas, siendo de esta manera igual al tratamiento alimento para ponedoras. También se observó que el efecto asociado a la semana presentó un aporte significativo, donde al pasar las semanas hay un incremento promedio de $3.051 \mathrm{~mm}$ del largo de la larva.

\section{DISCUSIÓN}

Los resultados obtenidos pueden deberse al valor nutricional de las dietas evaluadas, el alimento de ponedoras es un alimento de buen valor nutricional y de alta digestibilidad, presentando un contenido de proteína y carbohidratos adecuada para la alimentación de las larvas. La gallinaza de piso por el proceso de compostaje que sufre durante el periodo que está en el galpón (50 semanas) es enriquecida microbiológicamente, lo que aporta nutrientes de buena calidad para las larvas. Por otro lado, la porcinaza representa la parte del alimento que no es digerido por el porcino, un monogástrico especializado, siendo de menor valor nutricional para la alimentación de las larvas (16).

En un estudio reciente se evaluó el efecto de la relación proteína/carbohidratos en la dieta de larvas de mosca soldado, llegando a la conclusión que tanto la proteína como los carbohidratos dietéticos tuvieron un efecto significativo sobre el peso fresco y seco de las larvas, pero la proteína dietética fue un indicador más fuerte del peso fresco y seco de las larvas que el carbohidrato dietético. La composición larval también fue influenciada por el tipo de alimento, con larvas más pesadas que producen significativamente más grasa que las más ligeras (17). En otra investigación se evaluaron nueve dietas con diferentes relaciones proteína/ carbohidrato $(\mathrm{P} / \mathrm{C})$, donde se encontró que la proteína, más que la relación $\mathrm{P} / \mathrm{C}$ tiene influencia en el desarrollo, rendimiento larval, la grasa cruda larval y el rendimiento del huevo (18). 
Con los resultados obtenidos y la revisión de literatura se determina que la gallinaza y el alimento para ponedoras son una buena alternativa para el crecimiento de las larvas de mosca soldado, esto tal vez debido al contenido proteico de dichas dietas.

En conclusión por medio de los modelos mixtos de coeficientes aleatorios se determina que las larvas alimentadas con alimento comercial de ponedoras y gallinaza, no presentan diferencias significativas. Además, se observó que estos dos tipos de dieta son más eficientes en términos de crecimiento al comparar con porcinaza. En términos generales, se presentó mejor desempeño en peso, largo y ancho, en las larvas alimentadas con gallinaza y alimento para ponedoras. Sin embargo, es importante seguir realizando estudios que comprueben dichos resultados.

En la práctica se validaron los supuestos de homogeneidad de varianza y normalidad en los errores de los tres modelos mixtos utilizando pruebas gráficas para los residuales (Figura 4, 5 y 6), se observó buen ajuste en los modelos. Sin embargo, para el modelo de la variable Largo, se recomienda probar otro tipo de distribuciones que puedan modelar de forma más adecuada el comportamiento de dicha variable, puesto que el gráfico para la normalidad de los residuos (Figura 6), indica que hay ciertos datos que hacen que no se cumpla dicho supuesto, además, los residuales presentaron una distribución asimétrica. Se recomienda en estudios posteriores la utilización de modelos mixtos generalizados posiblemente con distribución gamma.
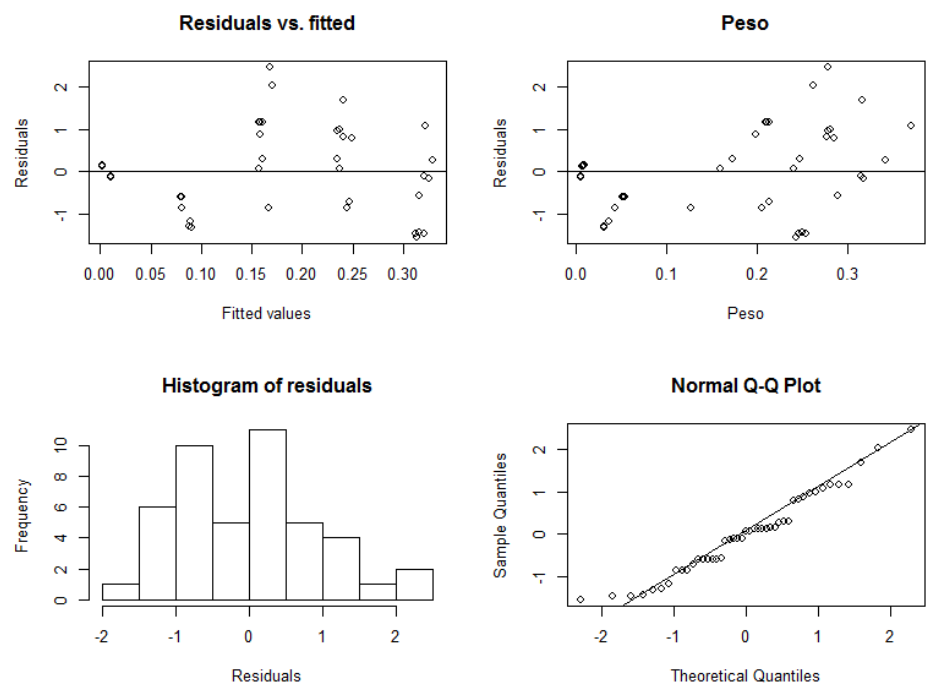

Figura 4. Gráficos para validar la homogeneidad de varianza y normalidad en el modelo para el peso
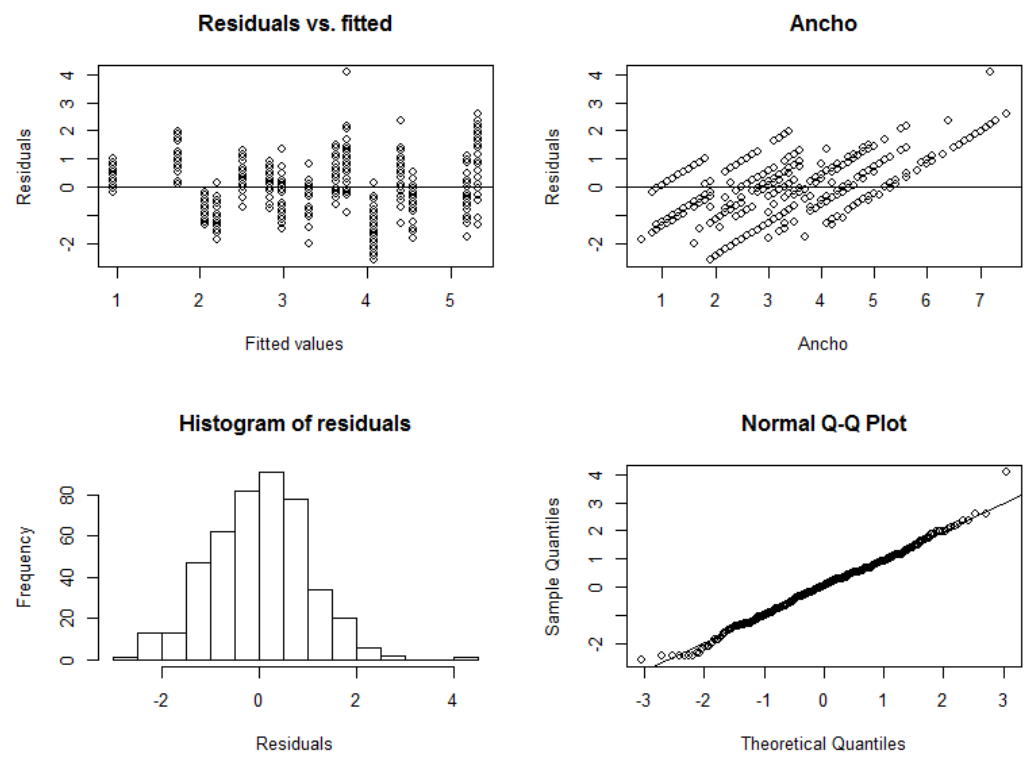

Figura 5. Gráficos para validar la homogeneidad de varianza y normalidad en el modelo para el ancho 

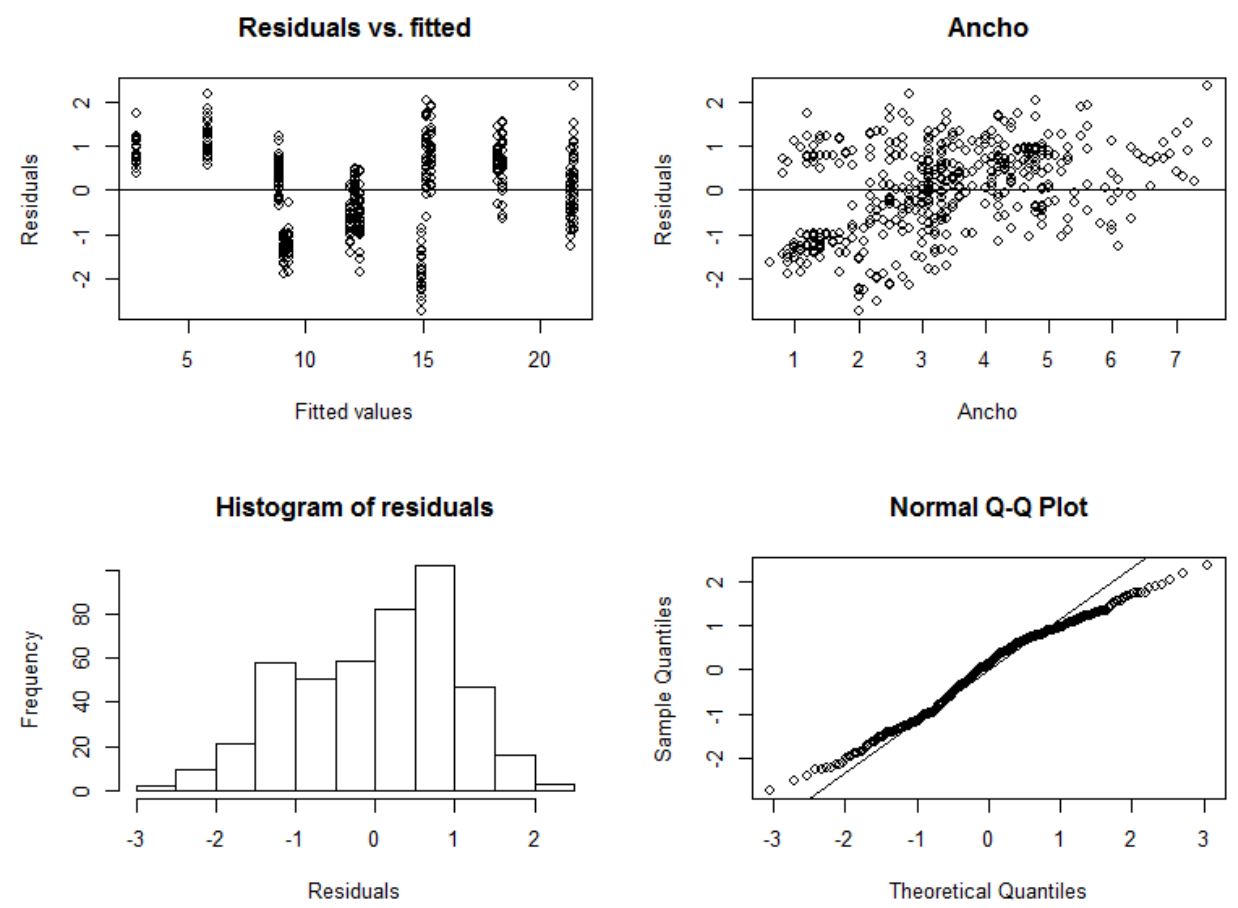

Figura 6. Gráficos para validar la homogeneidad de varianza y normalidad en el modelo para el largo

\section{Conflicto de intereses}

Los autores declaran que no tienen conflictos de interés con relación al tema de investigación del presente experimento.

\section{Agradecimientos.}

Al Centro de Biotecnología Industrial, CBI, SENA, Palmira, por facilitar las instalaciones para la realización del proyecto.

\section{REFERENCIAS}

1. FAO. La agricultura mundial en la perspectiva del año. Foro de expertos de alto nivel. Roma: FA0; 2009 . http:// www.fao.org/fileadmin/templates/wsfs/docs/Issues papers/Issues papers SP/La agricultura mundial.pdf

2. Dufour A, Bartram J. Animal waste, water quality and human health. World Health Organization: ROMA; 2012. https://www.who.int/water sanitation health/publications/animal waste chapters/en/

3. Marshall SA, Woodley NE, Hauser M. The historical spread of the black soldier fly, Hermetia illucens (L.) (Diptera, Stratiomyidae, Hermetiinae), and its establishment in canada. J Ent Soc Ont. 2015; 146:51-54. https://journal.lib. uoguelph.ca/index.php/eso/article/view/3696

4. Caruso D, Devic E, Subamia WI, Talamond P, Baras E. Technical handbook of domestication and production of diptera Black soldier fly (BFS) Hermetia illucens, Stratiomyidae. Penerbit IPB Press: Kencana JAVA; 2014. http:// horizon.documentation.ird.fr/exl-doc/pleins textes/divers17-11/010063336.pdf

5. Sheppard D, Newton G, Thompson SA. Value added manure management system using the black soldier fly. Bioresour Technol 1994; 50(3):275-279. https://eurekamag.com/research/002/556/002556823.php 
6. Barragan-Fonseca KB, Dicke M, van Loon JJA. Nutritional value of the black soldier fly (Hermetia illucens L.) and its suitability as animal feed: a review. J Insects Food Feed. 2017; 3(2):1-16. https://doi.org/10.3920/[IFF2016.0055

7. Sealey WM, Gaylord TG, Barrows FT, Tomberlin JK, McGuire MA, Ross C, Hilaire SSt. Sensory analysis of rainbow trout, Oncorhynchus mykiss, fed enriched black soldier fly prepupae, Hermetia illucens. J World Aquacult Soc. 2011; 42(1):34-45. https://doi.org/10.1111/j.1749-7345.2010.00441.x

8. Bondari K, Sheppard D. Soldier fly, Hermetia illucens L., larvae as feed for channel catfish, Ictalurus punctatus (Rafinesque), and blue tilapia, Oreochromis aureus (Steindachner). Aquacult. Res. 1987; 18(3):209-220. https:// doi.org/10.1111/j.1365-2109.1987.tb00141.x

9. Hale O. Dried Hermetia illucens larvae (Diptera: Stratiomyidae) as a feed additive for poultry. Ga Entomol Soc J. $1973 ; 8: 16-20$.

10. Van Huis A, Van Itterbeeck J, Klunder H, Mertens E, Halloran A, Muir G, Vantomme P Edible insects Future prospects for food and feed security. Roma: FAO Forestry; 2013. http://www.fao.org/docrep/018/i3253e/i3253e00.htm

11. Vargas E, Mata L. Utilización de las excretas de aves en la alimentación e rumiantes. Nutrición Animal Tropical. 1994; 1(1):59-71. https://revistas.ucr.ac.cr/index.php/nutrianimal/article/view/9979

12. Resolución 00150.Fertilizantesyacondicionadores de suelos. InstitutoColombianodeAgropecuario-ICA:Colombia; 2003. https://www.ica.gov.co/getattachment/1fb4d420-5e11-4ae6-959d-5bada49d683c/2003R0150-1.aspx

13. Rodríguez-Rodríguez GF, Sánchez-Camargo AM. Evaluación ambiental de los usos potenciales de la porcinaza posterior a su tratamiento de estabilización. Universidad de la Salle: Colombia; 2017. http://hdl.handle. net/10185/21739

14. Verbeke G. Linear mixed models for longitudinal data. In Linear mixed models in practice. Springer: New York, NY; 1997. https://www.springer.com/gp/book/9781441902993

15. Pinheiro J, Bates D, DebRoy S, Sarkar D, Heisterkamp S, Van Willigen B, Maintainer R. Package 'nlme'. Linear and Nonlinear Mixed Effects Models, version. 2017. https://CRAN.R-project.org/package=nlme

16. Oonincx DGA, Van Huis A, Van Loon JJA. Nutrient utilisation by black soldier flies fed with chicken, pig, or cow manure. J Insects Food Feed. 2015; 1(2):131-139. https://doi.org/10.3920/IIFF2014.0023

17. Beniers JJA, Graham RI. Effect of protein and carbohydrate feed concentrations on the growt and composition of black soldier fly (탈etia illucens) Larvae. J Insects Food Feed. 2019; 5(3):193-199. https://doi.org/10.3920/ ЏIFF2018.0001

18. Barragan-Fonseca KB, Gerrit G, Marcel D, van Loon JJA. Effects of dietary protein and carbohydrate on life-history traits and body protein and fat contents of the black soldier fly Hermetia illucens. Physiological Entomology. 2019; 44(2):148-159. https://doi.org/10.1111/phen.12285 